\title{
Chess Endgames and Neural Networks
}

\author{
Article
}

Published Version

Haworth, G. M. and Velliste, M. (1998) Chess Endgames and Neural Networks. ICGA Journal, 21 (4). pp. 211-227. ISSN 1389-6911 Available at https://centaur.reading.ac.uk/4569/

It is advisable to refer to the publisher's version if you intend to cite from the work. See Guidance on citing.

Published version at: http://ticc.uvt.nl/icga/journal/

Publisher: The International Computer Games Association

All outputs in CentAUR are protected by Intellectual Property Rights law, including copyright law. Copyright and IPR is retained by the creators or other copyright holders. Terms and conditions for use of this material are defined in the End User Agreement.

\section{www.reading.ac.uk/centaur}

\section{CentAUR}

Central Archive at the University of Reading

Reading's research outputs online 


\title{
CHESS ENDGAMES AND NEURAL NETWORKS
}

\author{
G.M C. Haworth ${ }^{1}$ and M. Velliste ${ }^{2}$ \\ Berkshire, England
}

\begin{abstract}
The existence of endgame databases challenges us to extract higher-grade information and knowledge from their basic data content. Chess players, for example, would like simple and usable endgame theories if such holy grail exists: endgame experts would like to provide such insights and be inspired by computers to do so. Here, we investigate the use of artificial neural networks (NNs) to mine these databases and we report on a first use of NNs on KPK. The results encourage us to suggest further work on chess applications of neural networks and other data-mining techniques.
\end{abstract}

\section{INTRODUCTION}

Endgame databases, particularly those of Ken Thompson (1986), have made a significant contribution to chess. Their existence and commercial availability invite the chess community to:

- re-analyse exemplar positions and games played over the board;

- validate, or 'cook' and possibly repair, published problems and studies;

- $\quad$ adjust past perceptions of and correct publications on various endgames, such as KQKR;

- $\quad$ identify new concepts, patterns and specific positions, such as zugzwangs and studies;

- create complete theories of particular endgames, such as KRKN and KBBKN;

- $\quad$ prioritise and simplify endgame theories to make them more usable for over-the-board games.

Nunn’s remarkable ground-breaking trilogy $(1992,1994,1995)$ analysed over twenty 'Thompson' profiles of force. It is a prime example of what can be achieved and illustrates all of the above. In a closing summary, Nunn expressed confidence (1995, p. 272) that endgames would continue to be explained "in more human friendly terms".

Such progress will be facilitated by the best possible symbiosis of man and machine, i.e., a combination of their respective capabilities of synthesis and analysis. If the symbiosis is enriched with good information presentation it will aid human cognition considerably (Roycroft and Beal, 1991). This contribution describes an exploration of neural-network data-mining techniques prompted by the second author's short degree-project on this technology. The KPK endgame was chosen as the application domain. The task was to demonstrate how a number of NNs learns to classify positions as wins or draws.

The neural network (NN) is a re-emerging technology. Samuel (1959) referred to NNs but Fürnkranz (1996) found few uses of NNs in computer chess and none on endgame databases. However, NNs have made significant contributions in the field of backgammon, perhaps because it is a game of both skill and chance. Tesauro $(1994,1995)$ taught NEUROGAMMON from existing games and then created the NN-based TDGAMMON which learned by playing itself. The latter is now (TD-GAMMON 3.0) the world's best backgammon program, rivals the human champions and has changed the theory of the game.

Section 2 describes the key aspects of the KPK domain and Section 3 those of NN technology. Section 4 describes the design of the NN experiment and Section 5 the training and testing of the NNs chosen. Finally, in Section 6 and 7, we suggest questions for further work and we put NNs in a broader data-mining context.

International Computers Limited, Sutton's Park, Reading, UK RG6 1AZ. Email: guy.haworth@icl.com.

Graduate, Cybernetics Department, The University of Reading, UK. 


\section{THE ENDGAME KPK}

Chess has been referred to as the fruit fly of artificial intelligence, an ideal laboratory subject with which to investigate new ideas (Michie, 1980). It provides:

- a synthetic, discrete, finite domain, known worldwide and defined by a few rules of the game;

- an almost static domain, those rules being controlled by FIDE and unlikely to change (although the rules of play which do not concern us here are more mutable);

- a clear definition of result - win, draw, or loss - against which new strategies may be tested;

- a body of past AI work with which any new ideas may be compared;

- a successful history of exercising AI initiatives in the past.

In addition, some 92 of the 145 three- to five-man chess endgames are now documented in basic data terms in databases which are:

- complete and essentially correct;

- of various known sizes, structured and exhibiting within them a range of problem-complexity

- $\quad$ populated by positions each with a defined value ('+', '=' or '-') and depth indications $(+n=$ White wins in $n$ ply, $0=$ draw, $-n=$ Black wins in $n$ ply $)$.

Of these 92 endgames, the KPK endgame has the following useful features. It:

- $\quad$ involves fewer positions than 4/5-man endgames by factors of $\approx 16 / 950$ and $\approx 60 / 3600$;

- comes with a useful set of concepts used to define principles of successful play;

- $\quad$ has been most used of all endgames to demonstrate a variety of approaches to endgame computer-play;

- features a significant proportion of draws as well as wins for White;

- includes positions with a range of complexity from trivial to deep and non-intuitive;

- $\quad$ is 'd-e axis' symmetric, halving the number of different positions.

\begin{tabular}{|c|c|c|c|c|c|c|}
\hline Set & Legal? & Piece positions & \# & + & $=$ & Notes \\
\hline 1 & Yes & $\{w K g 2 / h 2 / h 3, w P b 2, b K g 5:$ wtm $\}$ & 3 & 3 & 0 & Deepest wtm wins: 1. Kg3! 37 ply conv. \\
\hline 2 & Yes & $\{$ wKh3, wPb2, bKh5/h6: btm\} & 2 & 2 & 0 & Deepest btm wins: $1 . \ldots$ Kg5! 38 ply conv. \\
\hline 3 & Yes & $\{$ wKd1, wPc3, bKf8: wtm $\}$ & 1 & 1 & 0 & 1. Kc2! $1 . \mathrm{Kd} 2$ draws \\
\hline 4 & Yes & $\{\mathrm{wKd6}, \mathrm{wPb} 2, \mathrm{bKd} 3: \mathrm{wtm}\}$ & 1 & 1 & 0 & 1. Kd5! $1 . \mathrm{Kc} 5$ is sub-optimal \\
\hline 5 & Yes & $\{w K c 4$, wPb2, bKc8: wtm $\}$ & 1 & 1 & 0 & 1. Kb5! not 1. Kb4/Kc5 (sub-optimal) \\
\hline $6 a$ & no & $\{$ wKa6/b6/c6/c7/c8, wPa7, bKa8: wtm\} & 5 & 0 & 5 & Not reachable from the initial array \\
\hline $6 b$ & no & $\{\mathrm{wKx} 6, \mathrm{wPx} 7, \mathrm{bKx} 8: \mathrm{x}=\mathrm{b} / \mathrm{c} / \mathrm{d}: \mathrm{wtm}\}$ & 3 & 3 & 0 & Not reachable from the initial array \\
\hline $6 c$ & no & $\{$ wKc1/c2, wPa2, bKa1: wtm $\}$ & 2 & 2 & 0 & Not reachable from the initial array \\
\hline $6 d$ & no & $\{$ wKc7/c8, wPb6, bKa8: wtm $\}$ & 2 & 2 & 0 & Not reachable from the initial array \\
\hline $6 e$ & no & $\{$ wKa6/b6, wPc7, bKa8: wtm $\}$ & 2 & 2 & 0 & Not reachable from the initial array \\
\hline $7 a$ & no & $\{$ wK?, w'P'a8...d8, bK?: btm\} & 13,980 & 12,985 & 995 & Post-conversion positions not in KPK \\
\hline $7 b$ & no & $\{\mathrm{wK}$ ?, wPa2...d2, bKx3 in check: btm\} & 388 & 6 & 382 & not reachable from the initial array \\
\hline 7c & no & $\{$ wKa1, wPa2, bKc1/c2: btm $\}$ & 2 & 1 & 1 & not reachable from the initial array \\
\hline
\end{tabular}

Table 1: A selection of legal and illegal KPK positions.

Like any endgame, KPK can be characterised, for both wtm- and btm-positions in terms of its size, structure and profile. The total size of the domain and the \%-split of wtm/btm wins ('+') is clear (see Table 2) despite Clarke’s (1977) apparent 'one out' discrepancy. Some $76.5 \%$ of wtm-, 58.4\% of btm- and $67.3 \%$ of all KPK positions are won for White. Table 3 shows the number $\mathrm{N}_{\mathrm{p}}$ of 'won' wtm-positions convertible with best play in exactly $p$ ply.

The counts usually include a few illegal positions, which are unreachable from the original array (see Table 1) but are difficult to detect. For the record, we have subtracted the ones we know from Edwards' (1994) figures in Table 2 and Clarke's (1977) figures in Table 3. In Table 2, the figures presented are taken from Clarke (1977), Bramer (1980), Bramer (1982), Shapiro and Niblett (1982), Thompson (1986) and Edwards (1994). In the final column we have added our own findings. 


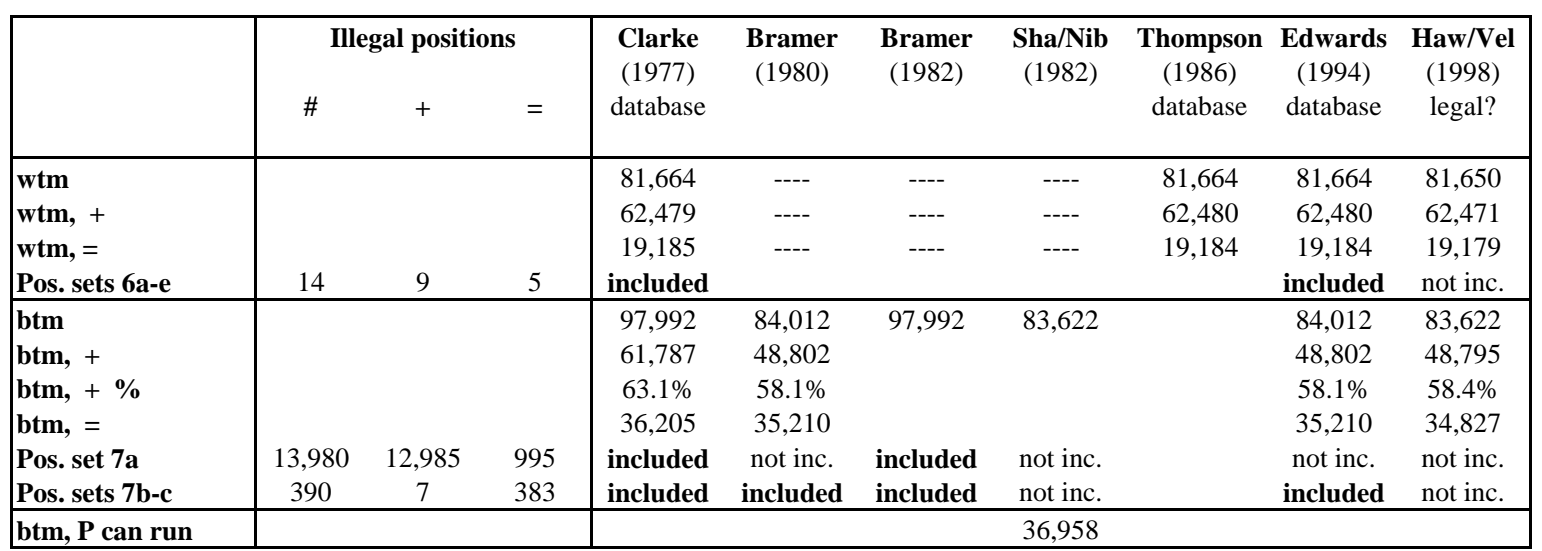

Table 2: Sources of numerical data on the KPK endgame.

The structure and profile of the KPK domain is indicated by the existence of subdomains of positions with specific characteristics. Examples relevant here include:

$\mathrm{S}=\{$ positions $\mathrm{p} \mid \mathrm{p} \in \mathrm{S} \Rightarrow$ 'best play' successors of $\mathrm{p}$ (before conversion) $\in \mathrm{S}\} \ldots$

let such subsets $\mathrm{S}$ be defined as 'closed'; $\mathrm{S}_{1}$ closed $\& \mathrm{~S}_{2}$ closed $\Rightarrow \mathrm{S}_{1} \cap \mathrm{S}_{2}$ closed;

two examples of such closed sets are used in this paper.

$\mathrm{C}_{\mathrm{p}}=\{$ won wtm positions $\mid$ won by conversion (P-promotion or mate) with best play in $\leq p$ ply $\}$

Clearly $\mathrm{C}_{1} \subset \mathrm{C}_{3} \subset \ldots \subset \mathrm{C}_{37}$ : $\mathrm{C}_{\mathrm{p}}$ is closed.

$\mathrm{F}_{\mathrm{x}}=\{\mathrm{wtm}$ positions $\mathrm{p} \mid$ Pawn on file $\mathrm{x}\}$

$\left\{\mathrm{F}_{\mathrm{x}}\right\}$ is a partition of KPK and $\mathrm{F}_{\mathrm{x}}$ is closed.

$\mathrm{P}_{\mathrm{q}}=\{$ positions $\mathrm{p} \mid$ Pawn on file $\mathrm{x} \geq \mathrm{q}\}$, regularly used in the KPK texts

also used in other endgames with a Pawn (Roycroft and Thompson, 1986; Nunn, 1992, 1995)

$\mathrm{P}_{\mathrm{q}}$ is closed and $\mathrm{P}_{7} \subset \mathrm{P}_{6} \subset \ldots \subset \mathrm{P}_{2}$. Therefore $\mathrm{C}_{\mathrm{p}} \cap \mathrm{F}_{\mathrm{x}} \cap \mathrm{P}_{\mathrm{q}}$ is closed.

$F_{x}$ is specific to KPK and generalises only to endgames featuring Pawns but the nested subsets $C_{p}$ are more interesting as they can be identified from any endgame database. Suppose an 'agent' (human or silicon) is to achieve a goal, such as $\mathrm{W}\left(\mathrm{C}_{9}\right)$, 'win any position in $\mathrm{C}_{9}$ '. It need only be able to effect a $\mathrm{C}_{9} \rightarrow \mathrm{C}_{7}$ transition and achieve $\mathrm{W}\left(\mathrm{C}_{7}\right)$. Further, the achievement of $\mathrm{W}\left(\mathrm{C}_{7}\right)$ might inform the achievement of the $\mathrm{C}_{9} \rightarrow \mathrm{C}_{7}$ transition and therefore of $\mathrm{W}\left(\mathrm{C}_{9}\right)$. W( $\left.\mathrm{C}_{1}\right)$ and $\mathrm{W}\left(\mathrm{C}_{3}\right)$ are clearly the first two of a sequence of ever more inclusive goals of escalating difficulty en route to $\mathrm{W}\left(\mathrm{C}_{37}\right)$.

This suggests that in general, if we want to train an agent to achieve goal $\mathrm{G}$ on some domain, it may be better to train it to walk rather than run first. The idea of training first on smaller closed subdomains and ascending to more difficult goals is revisited in Section 7.

Agents which secure wins over $C_{p}$ may be combined with a standard minimax program searching a tree to depth q to create a hybrid agent which wins over $\mathrm{C}_{\mathrm{p}+\mathrm{q}}$. The effectiveness of such an approach in KPK can be judged from Table 3 which shows the percentage $R_{p}$ of wtm wins not in $C_{p}$.

The complexity of KPK can be judged from both the chess and technology perspectives:

- $\quad 75.7 \%$ of White wins in btm-positions (Shapiro and Niblett , 1982, p. 81) involve only running the Pawn;

- Bramer (1980, p. 86) notes that even a USCF-2342 master missed optimal moves and a win;

- Table 1's positions 1-2 give the deepest wtm/btm wins with 37/38 ply to conversion respectively;

- Table 1's positions 3-5 illustrate other difficulties of securing the win and playing optimally: in position 3, White's Kc2 rather than $\mathrm{Kd} 2$ advances the $\mathrm{K}$ and gains the opposition; in position 4, White's Kd5 stops a bK advance both on the P and to c8; Kc5 does not; in position 5, White should not postpone the wKb5/bKb7 confrontation; Kb4/Kc5 are sub-optimal;

- the successful completion of computer programs has proved remarkably difficult: a residue of positions repeatedly escaping classification by a growing set of rules. 


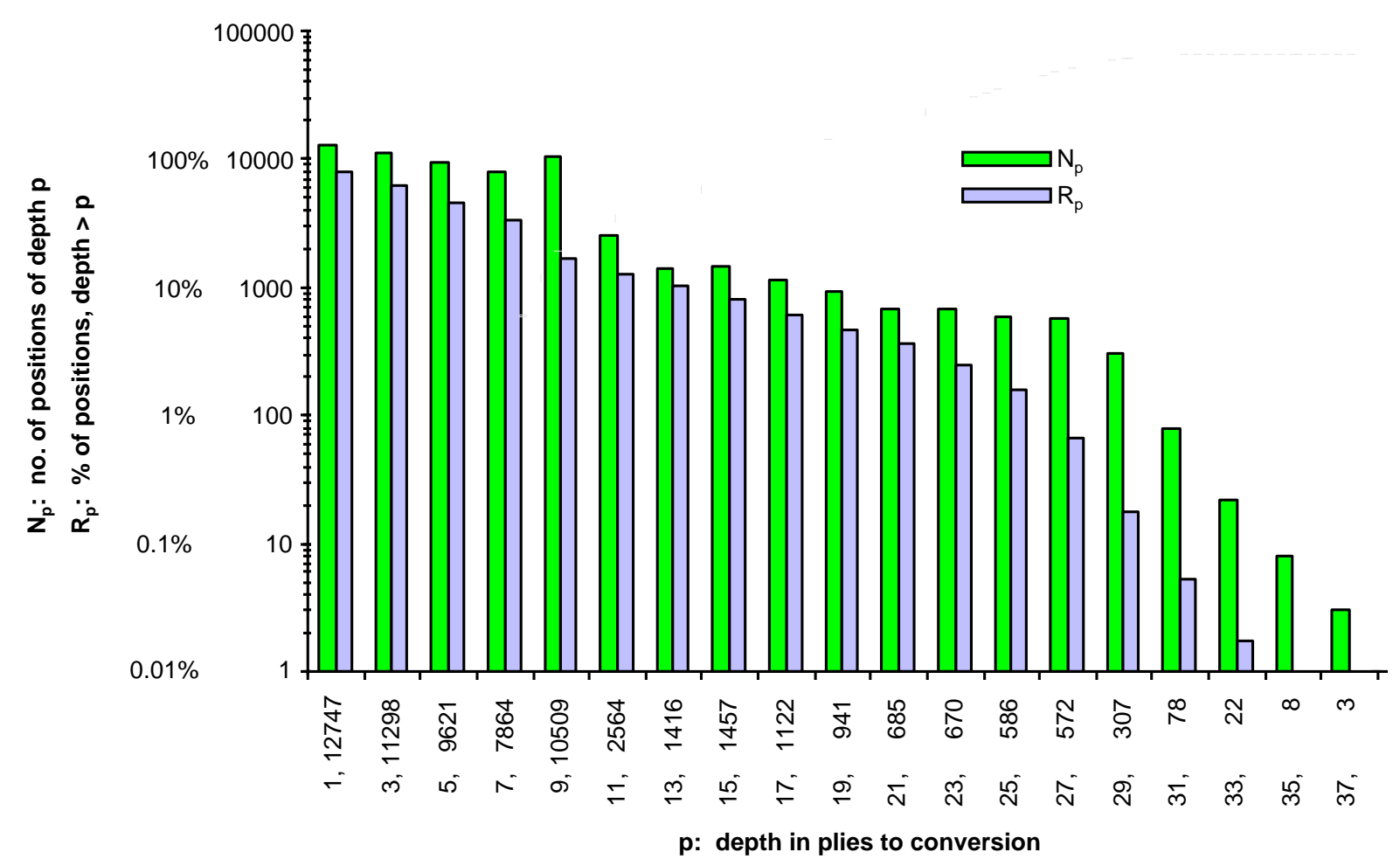

Table 3: wtm KPK wins: $\mathrm{N}_{\mathrm{p}}$ and $\mathrm{R}_{\mathrm{p}}$ for each (odd) ply-count $p$.

\section{NEURAL-NETWORK BASICS}

Figure 1 shows a multilayer perceptron (MLP), the particular type of NN chosen here. The MLP illustrated is a three-layer NN a-b-c-d as it has three layers of neurons. The general MLP is organised as $k$ layers of $N_{x}$ neurons each. To simplify nomenclature, the input nodes, which are not in fact neurons, are called input neurons in layer 0 . Each layer also has a notional neuron $\mathrm{N}_{0 \mathrm{x}}$ outputting ' 1 's (see the second row of Figure 1). Their weights $-t_{\mathrm{i}, \mathrm{x}+1}$ are used to define the input level at which the neurons threshold, that is, make their most rapid transition from 'output low' to 'output high'. The neurons in layers $1, \ldots, k-1$ are hidden neurons and those in the final layer ' $k$ ' are output neurons. Hence, Figure 1 distinguishes the input neurons, $\left(\mathrm{N}_{0 \mathrm{x}}\right)$,), the hidden neurons and the output neurons from each other.

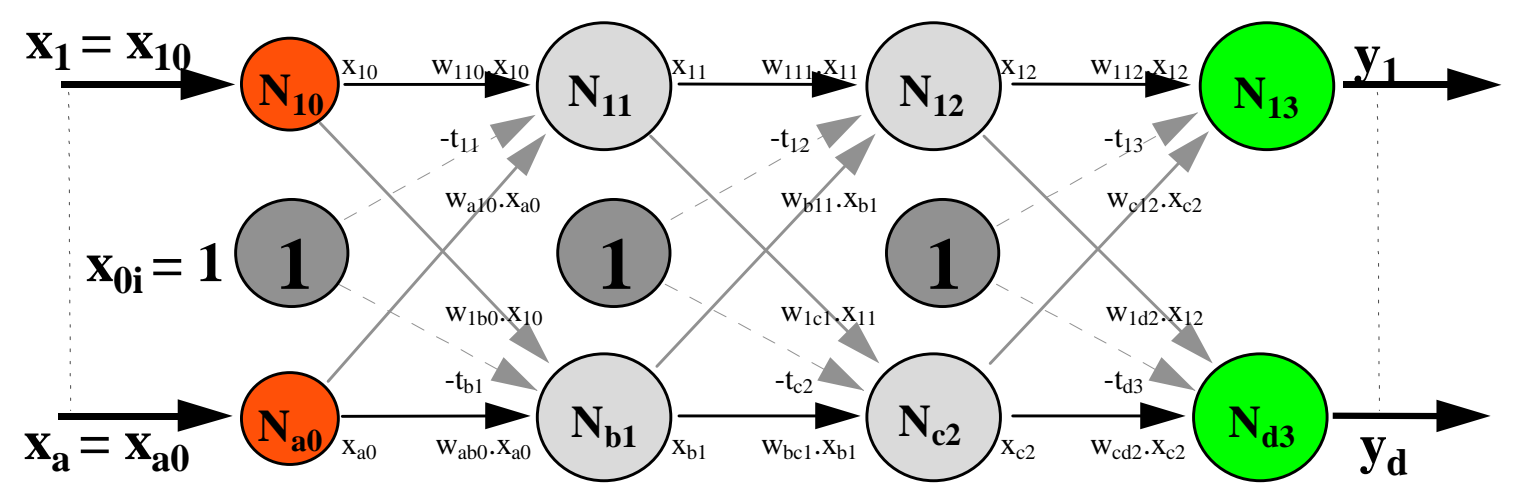

Figure 1: three-level MLP neural network a-b-c-d.

Representing the weights by $\underline{\mathbf{w}}$ and the inputs by $\underline{\mathbf{x}}$ with $\mathrm{x}_{0}=1$ (and all other parameters variable), the activation function for the neurons was taken to be the normal 'sum-squash' sigmoid function: 


$$
\begin{aligned}
& \mathrm{y}=\mathrm{f}(\underline{\mathbf{w}} \cdot \underline{\mathbf{x}})=\mathrm{f}(\mathrm{z})=1 /\left(1+\mathrm{e}^{-\mathrm{bz}}\right) \text { with } \mathrm{b}>0 . \\
& \text { Therefore, } \mathrm{f}(\mathrm{z}) \in(0,1), \mathrm{f}(0)=0.5, \mathrm{f}(\infty)=1, \mathrm{f}(-\infty)=0 \text { and } \mathrm{f}(\mathrm{z})+\mathrm{f}(-\mathrm{z})=1, \\
& \mathrm{f}^{\prime}(\mathrm{z})=\text { b.e } \mathrm{e}^{-\mathrm{bz}} /\left(1+\mathrm{e}^{-\mathrm{bz}}\right)^{2}=\mathrm{b} . \mathrm{f}(\mathrm{z}) \cdot \mathrm{f}(-\mathrm{z}) \\
& =
\end{aligned}
$$

As $\mathrm{b} \rightarrow \infty$, the function $\mathrm{f}$ tends to the Heaviside step function. In this paper $\mathrm{b}=1$.

A fully-connected $\mathrm{NN}$ has every one of the $\sum_{\mathrm{x}} \mathrm{N}_{\mathrm{x}}{ }^{*} \mathrm{~N}_{\mathrm{x}+1}$ possible connections made: neuron $\mathrm{N}_{\mathrm{ix}}$ in layer ' $\mathrm{x}$ ' transmits its output with weight $\mathrm{w}_{\mathrm{ij} \mathrm{x}}$ to $\mathrm{N}_{\mathrm{j}, \mathrm{x}+1}$ in the layer ' $\mathrm{x}+1$ ' only. It may be that many of these connections are not significant and can be pruned out without loss of NN functionality. This speeds up training and improves the chances of inferring higher level rules of classification from the NN after the training phase.

A neural network requiring $\mathrm{W}$ weights each of $\mathrm{B}$ storage bits requires $\mathrm{W} . \mathrm{B}+\varepsilon$ bits to represent it and this storage requirement may be compared with other ways of storing the function it performs to give a compression factor. Such an NN can be in only one of $2^{\text {W.B }}$ states and can therefore only represent, with a given characterisation and coding of input and output, at most one of $2^{\text {W.B }}$ functions.

The pure gradient descent version of the back-propagation training procedure BPT (Rich and Knight, 1991) may be parallelised on multi-processor computers and reads:

set the weights initially at random to values in the $[-0.1,+0.1]$ range

select a training set $\mathrm{T}$ of position characterisations $\left\{\underline{\mathbf{x}}_{i}\right\}$ with which to train the MLP

start_loop: in one sequence or epoch of training ...

input each of the vectors $\underline{\mathbf{x}}_{\mathrm{i}}$ in $\mathrm{T}$ and store the output vector $\mathbf{y}_{\mathrm{i}}$

after completing the epoch of training:

compare the outputs $\left\{\mathbf{y}_{\mathrm{i}}\right\}$ with the goal values $\left\{\underline{\mathbf{z}}_{\mathrm{i}}\right\}$, calculating $\left\{\mathbf{y}_{\mathrm{i}}-\underline{\mathbf{z}}_{\mathrm{i}}\right\}$

note how many of the instances in $\mathrm{T}$ are correctly classified

decide whether another epoch of training is required and if not, terminate

adjust the NN's weights $\underline{\mathbf{w}}$ to converge the outputs $\left\{\mathbf{y}_{\mathrm{i}}\right\}$ on goals $\left\{\underline{\mathbf{z}}_{\mathrm{i}}\right\}$

loop to start_loop for the next epoch of training.

The original perceptron (Minsky and Papert, 1969) has just one layer and one neuron. It can rank objects, positions in this case, in the same order as a single linear weighted sum (SLWS) $\underline{\mathbf{w}}$.... Such SLWSs were proposed and implemented by Samuel (1959) in checkers and are commonly used as the position evaluation functions in game programs. If a set of positions can be classified correctly into two subsets by the sign of the linear form $\underline{\mathbf{D}} . \underline{\mathbf{x}}$, a perceptron's weights $\underline{\mathbf{w}}$ can be iterated sufficiently close to $\underline{\mathbf{D}}$ to enable the perceptron to classify the positions correctly as well.

However, it is likely that the evaluation of positions using one linear function of their characteristics gives only an approximate guide to their correct ranking. Samuel (1967) concluded that an SLWS was too restricted to evaluate checkers positions effectively. Berliner (1980) improved the evaluation function of BKG 9.8, an early backgammon program, by upgrading from a SLWS to multiple LWS's (MLWS) and then, with more success, to a non-linear function. BKG 9.8 was the first program to defeat a World Champion at any board or card game. Tesauro, who later worked on DEEPER BLUE, also credits the shift from SLWS to non-linear NNs for the major improvements in his backgammon programs. The Othello program LOGISTELLO (Buro, 1995) was improved by changing from one SLWS to the next every four moves. Perhaps we should not be surprised that, for games requiring the effective combination of forces, position evaluation might involve non-linear evaluation of features on the board.

The study of perceptrons stalled when it was discovered (Minsky and Papert, 1969) that they had severe limitations in 'non-linear situations'. Their inability to emulate the XOR logic function is often illustrated in the textbooks. Fortunately, the generalisation of the single-layer perceptron to the MLP network and the discovery of the back-propagation training (BPT) method for MLPs revived the application of NNs (Rumelhart, Hinton and Williams, 1987; Beale and Jackson, 1990). Note that the neurons' activation function must be non-linear if the MLP is to react in a non-linear way to its inputs.

The increased adoption of non-linear functions coupled with the ability of MLPs to emulate such functions may offer a way forward for computer chess and other computer-played games. For example, any existing linear function $\underline{\mathbf{m}} . \underline{\underline{x}}$ used for evaluating chess positions on $n$ characteristics can be used to set up the initial 
position for training of not only an $n-1$ perceptron as above but of a potentially more subtle $n-n-1$ NN. In the notation above, the initialisation should be $\mathrm{w}_{\mathrm{ii} 0}=0.1, \mathrm{w}_{\mathrm{ij} 0} \approx \varepsilon^{2}(\mathrm{i} \neq \mathrm{j})$ and $\mathrm{w}_{\mathrm{i} 11}=\varepsilon \mathrm{m}_{\mathrm{i}}$ where $\varepsilon<<\mathrm{m}_{\mathrm{i}}$ and suitably small.

\section{THE DESIGN OF THE NEURAL-NETWORK EXPERIMENT}

A number of decisions needed to be made about:

- the goal $\boldsymbol{G}$ of the NN experiments on KPK,

- the characterisation $\boldsymbol{C}$ of KPK positions,

- the coding $\boldsymbol{I}$ of the characterisation as input to the NNs,

- the coding and interpretation $\boldsymbol{O}$ of the output,

- the number and size of the hidden layers $\boldsymbol{H}$,

- the choice of training sets $\boldsymbol{T}$ and testing sets $\boldsymbol{S}$, and

- the management $\boldsymbol{M}$, including termination decision, of the training phase.

Therefore, a NN experiment actually has parameters (G, C, I, O, H, T, S, M). The experimental space is indeed a large and complex one with a few navigational aids.

\subsection{The Characterisation of KPK Positions}

The experimental goal chosen here was to demonstrate NNs learning by training and to show to what extent the chosen NNs could classify wtm- or btm-KPK positions correctly into wins and draws. The classification of wtm and btm positions was seen as two tasks requiring distinct NNs.

The characterisation of the positions had to be adequate for the chosen goal and in terms of features which were computable. The characterisation should ideally lead to the explanation of concise classification rules if these exist.

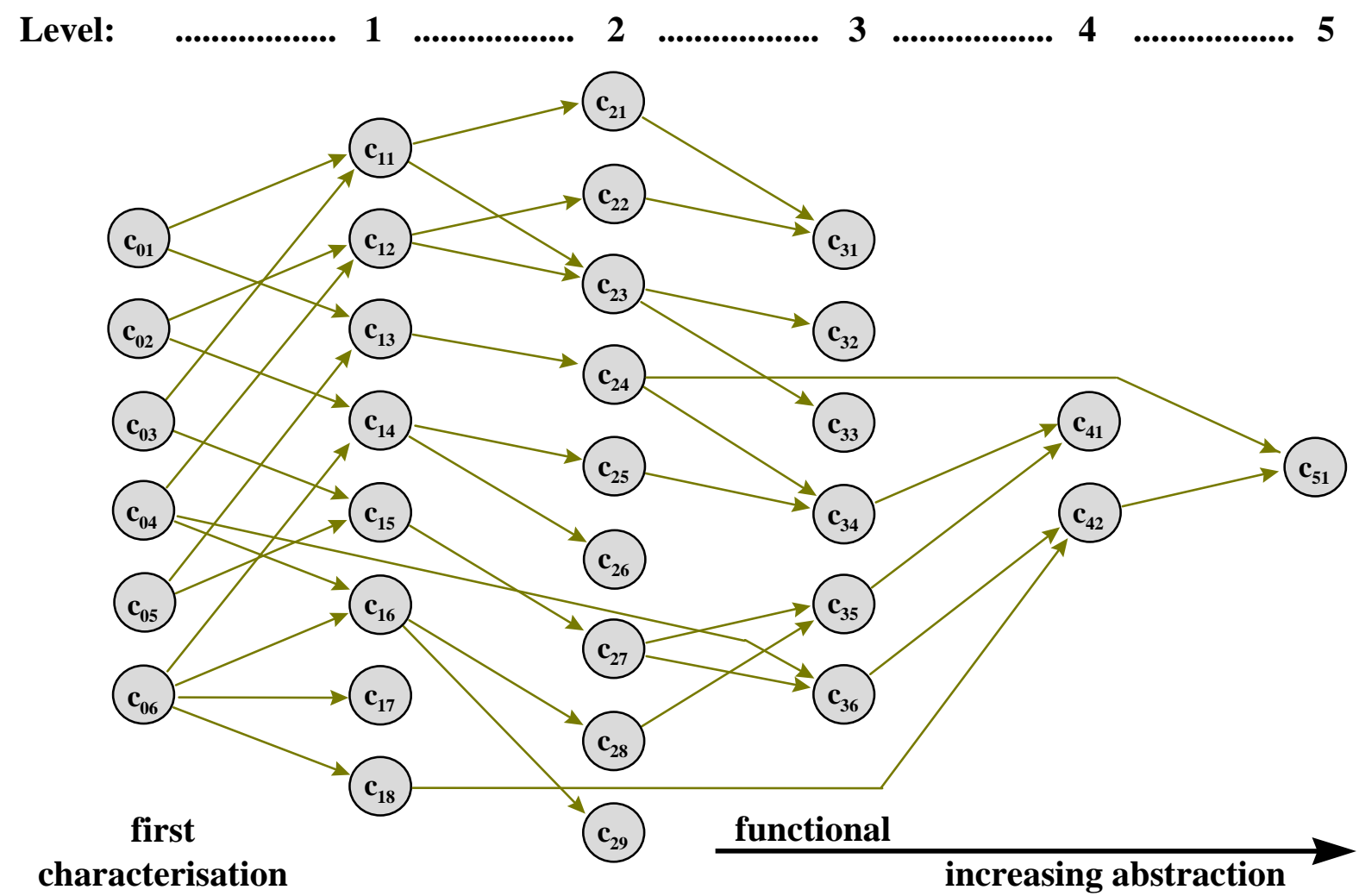

Figure 2: The lattice of characteristics and levels of abstraction. 
An inadequate characterisation by definition makes correct classification impossible: apples and oranges cannot be distinguished for example on the basis of size and weight. The physical characterisation of KPK using only the physical positions of the pieces is certainly adequate. However, the theory of KPK is discussed in more abstract concepts such as relative position, relative distance, key squares and opposition. Such abstractions are useful because they capture in a compact way the essence of some feature while suppressing unnecessary details. Further, past KPK knowledge representations (Beal, 1980; Bramer, 1980, 1982; Shapiro and Niblett, 1982) incorporated these abstractions in a large number of arithmetical rules which no doubt frustrated their authors with their increasing complexity and decreasing applicability.

There is also plenty of evidence from other games (Buro, 1995; Tesauro, 1995) that concepts established in the theory of a game (1) enrich the basic physical characterisation, (2) expedite the NN training process and (3) facilitate the interpretation of the characterised data in an evaluation function.

The characterisation can be thought of as being built up in layers (Quinlan, 1979), later characteristics being functionally derived from earlier ones as illustrated in Figure 2. The rule of assigning layer numbers $\mathrm{L}$ (c) to the characteristics ' $c$ ' is here:

$$
\mathrm{L}(\mathrm{c}) \geq 0 \text {; } \mathrm{L}(\mathrm{c}) \text { is minimised subject to constraint ' } \mathrm{c}=\mathrm{f}\left(\mathrm{c}_{1}, \ldots, \mathrm{c}_{\mathrm{n}}\right) \Rightarrow \mathrm{L}(\mathrm{c})>\mathrm{L}\left(\mathrm{c}_{\mathrm{i}}\right) \text { ' }
$$

If for example, $c_{2}=\left|c_{1}\right|$ it is clear that $L\left(c_{2}\right)>L\left(c_{1}\right)$. But if $c_{3}=c_{1}-c_{2}$, then $c_{1}=c_{2}+c_{3}$ and $c_{2}=c_{1}-c_{3}$. Therefore, one of $c_{1}, c_{2}$ or $c_{3}$ must be chosen to be a function of the other two $c_{i}$.

\begin{tabular}{|c|c|c|c|c|c|c|}
\hline Level & Char. & Description & $\mathbf{x}_{\mathbf{i}}$ & Formula & Range & $\mid$ Range \\
\hline 0 & $\mathrm{C}_{01}$ & $f(w K)$ : file of $w K$ & $\mathrm{x}_{1}$ & $\mathrm{X}_{1}$ & {$[1,8]$} & 8 \\
\hline 0 & $\mathrm{C}_{02}$ & $\mathrm{r}(\mathrm{wK})$ : rank of wK & $\mathrm{X}_{2}$ & $\mathrm{X}_{2}$ & {$[1,8]$} & 8 \\
\hline 0 & $\mathrm{C}_{03}$ & f(bK): file of bK & $\mathrm{x}_{3}$ & $\mathrm{x}_{3}$ & {$[1,8]$} & 8 \\
\hline 0 & $\mathrm{C}_{04}$ & $\mathrm{r}(\mathrm{bK})$ : rank of bK & $\mathrm{x}_{4}$ & $\mathrm{x}_{4}$ & {$[1,8]$} & 8 \\
\hline 0 & $\mathrm{C}_{05}$ & $f(w P)$ : file of $w P$ & $\mathrm{x}_{5}$ & $\mathrm{X}_{5}$ & {$[1,4]$} & 4 \\
\hline 0 & $\mathrm{C}_{06}$ & $r(w P)$ : rank of wP & $\mathrm{x}_{6}$ & $\mathrm{X}_{6}$ & {$[2,7]$} & 6 \\
\hline 1 & $\mathrm{c}_{11}$ & fd(wK, bK): wK-bK file difference & $\mathrm{x}_{7}$ & $\mathrm{X}_{1}-\mathrm{X}_{3}$ & {$[-7,7]$} & 15 \\
\hline 1 & $\mathrm{C}_{12}$ & rd(wK, bK): wK-bK rank difference & $\mathrm{x}_{8}$ & $\mathrm{x}_{2}-\mathrm{x}_{4}$ & {$[-7,7]$} & 15 \\
\hline 1 & $\mathrm{C}_{13}$ & $\mathrm{fd}(\mathrm{wK}, \mathrm{wP}):$ wK-wP file difference & $\mathrm{X}_{9}$ & $\mathrm{x}_{1}-\mathrm{x}_{5}$ & {$[-3,7]$} & 11 \\
\hline 1 & $\mathrm{C}_{14}$ & rd(wK, wP): wK-wP rank difference & $\mathrm{x}_{10}$ & $\mathrm{x}_{2}-\mathrm{x}_{6}$ & {$[-6,6]$} & 13 \\
\hline 1 & $\mathrm{C}_{15}$ & fd(bK, wP): bK-wP file difference & $\mathrm{x}_{11}$ & $\mathrm{x}_{3}-\mathrm{x}_{5}$ & {$[-3,7]$} & 11 \\
\hline 1 & $\mathrm{C}_{16}$ & rd(bK, wP): bK-wP rank difference & $\mathrm{X}_{12}$ & $\mathrm{x}_{4}-\mathrm{x}_{6}$ & {$[-6,6]$} & 13 \\
\hline 1 & $\mathrm{C}_{17}$ & $\mathrm{r}(\mathrm{wP})=8$ ? & $\mathrm{x}_{13}$ & $0 ; \mathrm{x}_{6}=8 \Rightarrow 1$ & {$[-6,6]$} & 13 \\
\hline 1 & $\mathrm{C}_{18}$ & no. of P-moves needed to convert P & $\mathrm{X}_{14}$ & $8-x_{6} ; x_{6}=2 \Rightarrow 5$ & {$[1,5]$} & 5 \\
\hline 2 & $\mathrm{C}_{21}$ & abs fd(wK, bK) & $\mathrm{X}_{15}$ & $\left|\mathrm{x}_{7}\right|$ & {$[0,7]$} & 8 \\
\hline 2 & $\mathrm{c}_{22}$ & abs rd(wK, bK) & $\mathrm{X}_{16}$ & $\left|\mathrm{x}_{8}\right|$ & {$[0,7]$} & 8 \\
\hline 2 & $\mathrm{C}_{23}$ & parity re 'opposition' & $\mathrm{X}_{17}$ & $0 ; x_{7}$ even $\& x_{8}$ even $\Rightarrow 1$ & {$[0,1]$} & 2 \\
\hline 2 & $\mathrm{C}_{24}$ & abs fd(wK, wP) & $\mathrm{X}_{18}$ & $\left|\mathrm{x}_{9}\right|$ & {$[0,7]$} & 8 \\
\hline 2 & $\mathrm{C}_{25}$ & abs $\operatorname{rd}(w K, w P)$ & $\mathbf{X}_{19}$ & $\left|\mathrm{x}_{10}\right|$ & {$[0,6]$} & 7 \\
\hline 2 & $\mathrm{C}_{26}$ & wK behind wP? & $\mathrm{X}_{20}$ & $0 ; \mathrm{x}_{10}<0 \Rightarrow 1$ & {$[0,1]$} & 2 \\
\hline 2 & $\mathrm{C}_{27}$ & abs fd(bK, wP) & $\mathrm{X}_{21}$ & $\left|\mathrm{x}_{11}\right|$ & {$[0,7]$} & 8 \\
\hline 2 & $\mathrm{C}_{28}$ & abs rd(bK, wP) & $\mathrm{X}_{22}$ & $\left|\mathrm{x}_{12}\right|$ & {$[0,6]$} & 7 \\
\hline 2 & $\mathrm{C}_{29}$ & bK behind wP? & $\mathrm{X}_{23}$ & $0 ; \mathrm{x}_{12}<0 \Rightarrow 1$ & {$[0,1]$} & 2 \\
\hline 3 & $\mathrm{C}_{31}$ & d(wK, bK): wK-bK 'distance' & $\mathrm{X}_{24}$ & $\max \left(\mathrm{x}_{15}, \mathrm{x}_{16}\right)$ & {$[2,7]$} & 6 \\
\hline 3 & $\mathrm{c}_{32}$ & Wh. 'may have opposition' & $\mathrm{X}_{25}$ & $0 ; \mathrm{wtm} / \mathrm{x}_{17}=0$ or btm/ $\mathrm{x}_{17}=1 \Rightarrow 1$ & {$[0,1]$} & 2 \\
\hline 3 & $\mathrm{C}_{33}$ & Bl. 'may have opposition' & $\mathrm{x}_{26}$ & $0 ; \mathrm{wtm} / \mathrm{x}_{17}=1$ or btm/ $\mathrm{x}_{17}=0 \Rightarrow 1$ & {$[0,1]$} & 2 \\
\hline 3 & $\mathrm{c}_{34}$ & d(wK, wP): wK-wP 'distance' & $\mathrm{X}_{27}$ & $\max \left(\mathrm{x}_{18}, \mathrm{x}_{19}\right)$ & {$[1,7]$} & 7 \\
\hline 3 & $\mathrm{C}_{35}$ & d(bK, wP): bK-wP 'distance' & $\mathrm{X}_{28}$ & $\max \left(\mathrm{x}_{21}, \mathrm{x}_{22}\right)$ & {$[1,7]$} & 7 \\
\hline 3 & $\mathrm{C}_{36}$ & 'distance', bK to wP's queening sq. & $\mathbf{X}_{29}$ & $\max \left(\mathrm{x}_{21},\left|8-\mathrm{x}_{4}\right|\right)$ & {$[0,7]$} & 8 \\
\hline 4 & $\mathrm{C}_{41}$ & $\mathrm{~d}(\mathrm{wK}, \mathrm{wP})-\mathrm{d}(\mathrm{bK}, \mathrm{wP})$ & $\mathrm{X}_{30}$ & $\mathrm{X}_{27}-\mathrm{X}_{28}$ & {$[-6,6]$} & 13 \\
\hline 4 & $\mathrm{C}_{42}$ & $\mathrm{~d}(\mathrm{bK}, \mathrm{Qs})-\mathrm{m}(\mathrm{P}, \mathrm{Qs})$ & $\mathrm{x}_{31}$ & $\mathrm{X}_{29}-\mathrm{X}_{14}$ & {$[-5,6]$} & 12 \\
\hline 5 & $\mathrm{C}_{51}$ & P can run and convert? & $\mathrm{X}_{32}$ & $\mathrm{x}_{18}>0 \& \mathrm{x}_{31}>1(\mathrm{btm}, 2) \Rightarrow 1$ & {$[0,1]$} & 2 \\
\hline
\end{tabular}

Table 4: Input format and characterisation of KPK. 
The chosen characterisation, shown in Figure 2 and Table 4, was compiled as a practical subset of the available theory and included the following features:

- relative distances of the black King and Pawn from the Pawn's 'queening' square,

- $\quad$ rank and file positions of the Kings relative to the Pawn, and

- 'possible opposition' indicators based on the parity of the Kings' relative rank and file positions.

All characteristics were included in the chosen characterisation if they were en route to any characteristic that was also included. Note that this is not always necessary; had the board had an infinite number of files, $x_{1}, x_{3}$ and $\mathrm{x}_{5}$ would have been irrelevant. It was not necessary to indicate which side had the move as an individual NN was being presented with either wtm- or btm-positions.

Positions which were clearly illegal or known to be unreachable (see Table 1) were not included in the training and testing sets. In this spirit, a belated decision was taken not to follow Clarke (1977) who included positions with the Pawn on the $8^{\text {th }}$ rank. Input $\mathrm{x}_{13}$ therefore became redundant and could have been removed. Similarly, one of $\mathrm{x}_{25}$ and $\mathrm{x}_{26}$ is redundant but both would have been required if a closer approximation to the concept of opposition had been used which occasionally credited neither side with having the opposition.

The concept 'Kings on opposite sides of the Pawn' was not included; example 3 of Table 1 suggests that it might also have been useful. Nor did the characterisation emphasise 'Pawn on $2^{\text {nd }}$ rank' or 'King distances from c8' in the context of the Pawn being on the a-file.

\subsection{Coding the Characterisation of Positions}

The 'natural' coding was chosen for the KPK NNs, each input taking the values indicated under 'range' in Table 4. There was more than one alternative, as illustrated below, but these seemed to have no compelling advantages.

A binary coding of the input could have been used with for example $c_{11}$ ( $x_{7}$ in Table 4 ) being represented by 4 binary inputs covering the range $0-14$. The win/draw classification problem using for example just the six basic characteristics $\mathrm{C}_{01}-\mathrm{C}_{06}$ then becomes one of emulating a Boolean function on 17 Boolean variables.

The reduction of the classification problem to one of emulating a Boolean function appears to be attractive, given a concise but little known result by Messom (1992) cited by Hinde et al. (1997):

any Boolean function on $n$ variables may be emulated by an $n-n-1 \mathrm{NN}$ with only $(n+1)^{2}$ weights

Although this result nominates an adequate NN and potentially removes the need to choose any other NN, a quick calculation shows that the price to be paid is in the precision of the weights. There are $2^{\mathrm{n}}$ values for a vector of $n$ Boolean variables and $2 \wedge 2^{\mathrm{n}}$ Boolean functions mapping these to \{false, true . Therefore, as $(n+1)^{2}$ weights of $\mathrm{B}$ bits can only be in one of $2^{\wedge}(n+1)^{2} \mathrm{~B}$ states, the number of storage bits required for at least some of the NNs' weights must be greater than $2^{n} /(n+1)^{2}$. This implies for example at least 42,73 and 405 bits when $n$ takes the values 13, 14 and 17. The chosen characterisation would have required 92 binary inputs.

Given a standard representation of weight numbers, the least possible value of the maximum number of bits required in any weight to emulate a particular Boolean function could act as the Boolean function's class number and possibly as its complexity index.

The Boolean coding of the characterisation was not chosen for two further reasons. First, it seems to lose the apparently essential arithmetical properties of 'order' and 'difference'. Second, it is not clear how quickly the $n-n-1$ network will train or whether it will reveal more readily any higher-level concepts of value when trained. 
Tesauro (1992a, Section 2.3) regarded this and the output coding as key decisions; his choice of characterisation coding for TD-GAMMON used neither of the 'natural' or 'binary' extremes. The number of white or black pieces on a point of the board was represented by eight inputs, four for each colour and 192 inputs in all. As pieces of only one colour are allowed on each point, at least one of these quartets would represent 'zero'. Each of the first three inputs (per colour) represented the absence/presence of the first, second and third pieces by $0 / 1$; the fourth input was 0 or $(n-3) / 2$ if there were more than 3 pieces on the point. Tesauro's aim seems to have been to keep the coding straightforward and the inputs mainly in the $[0,1]$ range.

\subsection{Coding Target Outputs; Interpreting Actual Outputs}

Two techniques were adopted here. Five of the six NNs used had just one output neuron while the last one had two outputs. The outputs $y_{i}$ are in the $(0,1)$ range (see Figure 1$)$.

For single-output NNs, the target values for $\mathrm{y}_{1}$ were ' 1 ' for a win and ' 0 ' for a draw. Actual outputs were always interpreted by ' $\mathrm{y}_{1} \geq 0.5$ indicates 'win'; $\mathrm{y}_{1}<0.5$ indicates 'draw' ”.

For the last NN, we followed the recommendation of providing an NN with $\mathrm{T}$ outputs if it is intended to classify objects into T types: the NN was given two outputs. Target values for $\left(y_{1}, y_{2}\right)$ were $(1,0)$ for a win and $(0,1)$ for a draw. Actual outputs were interpreted by the rule:

$$
\text { “ } \mathrm{y}_{1} \geq 0.5+\mathrm{m}, \mathrm{y}_{2}<0.5-\mathrm{m} \text { indicates 'win'; } \mathrm{y}_{1}<0.5-\mathrm{m}, \mathrm{y}_{2} \geq 0.5+\mathrm{m} \text { indicates 'draw' ”, } \mathrm{m}=0 / 0.4
$$

with the 0.4 value being used in the stopping condition for trials 38 to 41 in Table 5 . If $\mathrm{m}>0$, this allows an NN to 'pass' on a hybrid panel of advisors (Walker, 1996) on some occasions. Table 6 shows that the NN succeeded in producing symmetric outputs on $\mathrm{y}_{1}$ and $\mathrm{y}_{2}$ as requested.

Had the NN been trained not only on position value but on depth to win for won positions, the coding of target depth $d$ would have had to be decided. Perhaps $1-\left[\log _{e}(d+4) / 4\right]$ would have sufficed as depth in ply is less than 40 for KPK.

For training positions, the classification can be noted either as soon as the training position is encountered or at the end of an epoch of training. The experiments here did the latter.

\subsection{Choice of Hidden Topology}

Tesauro's TD-GAMMON has an NN with just two levels but some 40 to 160 hidden neurons (Sutton and Barto, 1998). However, there are few guidelines about the efficacy of 2-layer NNs and they are not sufficient to guarantee that a 2-layer NN will be effective for the chosen experimental goal. Messom's (1992) result is an existence proof only and says nothing about the required precision of the NN's weights.

Beale and Jackson (1990, pp. 84-85) note that, provided the characterisation is adequate:

- a 2-layer NN can adequately classify objects in a single convex hull in characterisation space, the convex hull being bounded by hyperplanes $\underline{\mathbf{w}_{\mathrm{i}}} \cdot \underline{\underline{\mathbf{x}}}=0(\mathrm{i}=1, \ldots, \mathrm{k})$;

- $\quad$ a set of $\mathrm{N}$ objects can be segregated from those objects not in the set by $\leq \mathrm{N}$ convex hulls;

- $\quad$ a 3-layer NN can classify N objects by OR-ing together outputs from $\leq$ N 2-layer NNs.

A decision was therefore made to adopt 3-layer NNs throughout rather than experiment with possibly limited 2-layer NNs. The decision cost is that 3-layer NNs take longer to train than 2-layer NNs.

The first and second layers of hidden neurons had $\mathrm{N}_{1}$ and $\mathrm{N}_{2}$ neurons with $\mathrm{N}_{1} \geq \mathrm{N}_{2}$ because the 'win subspace' was expected to involve more hyperplanes than convex hulls. The numbers chosen fairly arbitrarily probably varied from the parsimonious to the generous. Table 5 indicates in column 3 which NN was being used in any particular trial. 
The classification of wtm- and btm-positions was regarded as two separate problems. While a program interface to Thompson's KPK database was being developed, two small sets of respectively wtm- and btmpositions were hand prepared. These positions were either interesting 'borderline' ones from the texts or were generated at random. They were checked for legality and reachability and evaluated as wins or draws. These sets were then randomly divided into two sets, creating the training sets $T_{1}$ and $T_{2}$ and the testing sets $S_{1}$ and $S_{2}$, to ensure as far as possible that $T_{i}$ was characteristic of $S_{i}$. No position in $S_{i}$ is in $T_{i}$ to ensure that the positions used in the testing phase are all previously unseen.

Later, the Thompson KPK database yielded the three large sets $T_{3}, T_{4}$ and $T_{5}$. These sets were used in their entirety in the training mode. The complete collection of position sets was therefore:

$\begin{array}{ll}\mathrm{T}_{1} \text { and } \mathrm{T}_{2} & 125 \mathrm{wtm} \text { - and } 136 \mathrm{btm} \text {-positions respectively, } \\ \mathrm{S}_{1} \text { and } \mathrm{S}_{2} & \text { (the testing sets) } 125 \mathrm{wtm} \text { - and } 136 \text { btm-positions: } \mathrm{T}_{1} \cap \mathrm{S}_{1}=\mathrm{T}_{2} \cap \mathrm{S}_{2}=\varnothing, \\ \mathrm{T}_{3} & \text { all 81,664 wtm positions, } \\ \mathrm{T}_{4} & \mathrm{~F}_{\mathrm{a}} \text { (see Section 2): the 20,643 wtm positions with the wP on the a-file } \\ \mathrm{T}_{5} & \mathrm{~F}_{\mathrm{d}} \text { : the 20,346 wtm-positions with the wP on the d-file. }\end{array}$

The positions were presented in an arbitrary order which did not change from one training epoch to the next. Note that no attempt was made to present positions in any defined order of complexity.

With $\mathrm{y}_{\mathrm{ij}}$ as the actual output value and $\mathrm{d}_{\mathrm{ij}}$ as the target value of output neuron $\mathrm{j}$ to object $\mathrm{i}$, error-functions $\mathrm{E}_{1}$ and $E_{2}$ were defined as $E_{1}=$ average $\left|y_{i j}-d_{i j}\right|$ and $E_{2}=\max \left|y_{i j}-d_{i j}\right|$. Clearly, $E_{2}>E_{1}$.

Targets were set for the reduction of $E_{1}$ and $E_{2}$; these were variously $0.05,0.1$ and 0.15 . Training was terminated if, for the last 20 epochs of training, the error target had been exceeded or the NN had failed to improve the number of positions classified correctly.

Training was carried out using only the training set of positions and the trained network was then tested if necessary on the testing set of unseen positions. The training algorithm (Rich and Knight, 1991) was a variant of the 'pure gradient descent method' described previously and was as follows:

set the weights initially at random to init with values in the $[-0.1,+0.1]$ range,

start_loop: perform an epoch of training, presenting each input training set position in turn after presenting each position characterisation vector $\underline{\mathbf{x}}_{i}$ : compare the outputs $\underline{y}_{i}$ with the goal values $\underline{\mathbf{d}}_{i}$ adjust the NN's weights $\underline{\mathbf{w}}$ such that the output $\underline{y}_{i}$ would have been closer to $\underline{\mathbf{d}}_{i}$ (no use was made of the NN-training concepts of momentum and annealing)

at the end of an epoch of training, decide whether another epoch of training is needed: classify the positions of the training set using the end-epoch state of the NN ... noting the individual output errors $\left|\mathrm{y}_{\mathrm{ij}}-\mathrm{d}_{\mathrm{ij}}\right|$ for each position, (record the state of the NN in terms of its weights' values, $\mathrm{E}_{1 / 2}$ and \%-accuracy) consider whether the error measure $\mathrm{E}_{1 / 2}$ has been consistently below the error target $\mathrm{E}$, and consider whether the NN's success rate on the training set positions has apparently peaked if another epoch of training is required, return to start_loop if another epoch is not indicated, choose the end-of-epoch $\mathrm{NN}$-state with the minimal $\mathrm{E}_{1 / 2}$

The training process is effectively 'programming by example’. It replaces the intellectual process of deciding how to apply a set of identified rules either manually (Beal and Clarke, 1980; Bramer, 1980) or with a degree of automation (Quinlan, 1979; Bramer, 1982; Shapiro and Niblett, 1982).

Testing the network after the training phase merely involves presenting the positions of the testing set to the NN and interpreting the outputs as described in Section 4.3. 


\section{THE EXPERIMENTAL RESULTS}

Table 5 provides an overview of our experimental results. The column comp compares the memory requirements of a KPK database (taken to be $2^{17}$ bits) with the memory requirements to store the NNs' weights. An NN which effectively stores some data but with a smaller memory requirement has effectively compressed that data. Each weight was a 64-bit floating point number and no attempt was made to reduce the size or number of weights by pruning out small weights or rounding to 32-bit form.

\begin{tabular}{|c|c|c|c|c|c|c|c|c|c|c|c|c|}
\hline \multirow{2}{*}{$\begin{array}{cc}\text { Trial } & \text { wtm/ } \\
\# & \text { btm }\end{array}$} & \multicolumn{5}{|c|}{ Neural network } & \multirow{2}{*}{$\begin{array}{c}\text { Tr. } \\
\text { set } \\
\text { T }\end{array}$} & \multirow{3}{*}{$\begin{array}{c}\text { Target } \\
\text { Error } \\
\text { e }\end{array}$} & \multirow{3}{*}{$\begin{array}{c}\text { Epochs } \\
\text { taken }\end{array}$} & \multirow{2}{*}{\multicolumn{2}{|c|}{$\begin{array}{l}\text { Training } \\
\text { Accuracy, \% } \\
\text { correct }\end{array}$}} & \multirow{2}{*}{\multicolumn{2}{|c|}{$\begin{array}{l}\text { Testing } \\
\text { Accuracy, \% } \\
\text { correct }\end{array}$}} \\
\hline & \# & Topology & \# wts. & bits & comp. & & & & & & & \\
\hline \multicolumn{11}{|c|}{ For Error Function E1 } & & \\
\hline wtm & 1 & $32-10-10-1$ & 451 & 28,864 & 4.541 & 1 & 0.15 & 20 & 94 & 6 & 77 & 23 \\
\hline 2 & & & & & & 1 & & 29 & 92 & 8 & 74 & 26 \\
\hline 3 & & & & & & 1 & 0.10 & 39 & 98 & 2 & 82 & 18 \\
\hline 4 & & & & & & 1 & & 33 & 96 & 4 & 79 & 21 \\
\hline 5 & & & & & & 1 & 0.05 & 123 & 98 & 2 & 76 & 24 \\
\hline 6 & & & & & & 1 & & 99 & 98 & 2 & 77 & 23 \\
\hline 7 & & & & & & 1 & & 320 & 97 & 3 & 75 & 25 \\
\hline 8 & 2 & $32-20-10-1$ & 881 & 56,384 & 2.325 & 1 & 0.15 & 17 & 97 & 3 & 78 & 22 \\
\hline 9 & & & & & & 1 & 0.10 & 28 & 98 & 2 & 79 & 21 \\
\hline 10 & & & & & & 1 & 0.05 & 50 & 98 & 2 & 80 & 20 \\
\hline 11 & 3 & $32-32-5-1$ & 1,227 & 78,528 & 1.669 & 1 & 0.15 & 17 & 98 & 2 & 76 & 24 \\
\hline 12 & & & & & & 1 & 0.10 & 22 & 97 & 3 & 81 & 19 \\
\hline 13 & & & & & & 1 & 0.05 & 48 & 99 & 1 & 79 & 21 \\
\hline $14 \quad$ btm & 1 & $32-20-10-1$ & 881 & 56,384 & 2.325 & 2 & 0.15 & 37 & 92 & 8 & 82 & 18 \\
\hline 15 & & & & & & 2 & 0.10 & 48 & 97 & 3 & 83 & 17 \\
\hline 16 & & & & & & 2 & 0.05 & 130 & 98 & 2 & 87 & 13 \\
\hline 17 & & & & & & 2 & & 235 & 98 & 2 & 84 & 16 \\
\hline 18 & 3 & $32-32-5-1$ & 1,227 & 78,528 & 1.669 & 2 & 0.15 & 41 & 93 & 7 & 87 & 13 \\
\hline 19 & & & & & & 2 & 0.10 & 70 & 98 & 2 & 85 & 15 \\
\hline 20 & & & & & & 2 & 0.05 & 99 & 98 & 2 & 88 & 12 \\
\hline \multicolumn{13}{|c|}{ For Error Function E2 } \\
\hline $21 \quad$ wtm & 3 & $32-32-5-1$ & 1,227 & 78,528 & 1.669 & 1 & 0.10 & 90 & 98 & 2 & 77 & 23 \\
\hline 22 & & & & & & 1 & 0.10 & 53 & 100 & 0 & 79 & 21 \\
\hline 23 & & & & & & 1 & 0.10 & 113 & 100 & 0 & 81 & 19 \\
\hline 24 & 4 & $32-64-10-1$ & 2,773 & 177,472 & 0.739 & 1 & 0.10 & 60 & 100 & 0 & 77 & 23 \\
\hline 25 & & & & & & 1 & 0.10 & 67 & 99 & 1 & 79 & 21 \\
\hline 26 & & & & & & 1 & 0.10 & 60 & 99 & 1 & 77 & 23 \\
\hline 27 & 5 & $32-64-20-1$ & 3,433 & 219,712 & 0.597 & 1 & 0.10 & 49 & 100 & 0 & 74 & 26 \\
\hline 28 & & & & & & 1 & 0.10 & 48 & 100 & 0 & 76 & 24 \\
\hline $29 \quad$ btm & 3 & $32-32-5-1$ & 1,227 & 78,528 & 1.669 & 2 & 0.10 & 101 & 99 & 1 & 87 & 13 \\
\hline 30 & & & & & & 2 & 0.10 & 116 & 100 & 0 & 90 & 10 \\
\hline 31 & & & & & & 2 & 0.10 & 194 & 100 & 0 & 86 & 14 \\
\hline 32 & & & & & & 2 & 0.10 & 300 & 100 & 0 & 86 & 14 \\
\hline 33 & 4 & $32-64-10-1$ & 2,773 & 177,472 & 0.739 & 2 & 0.10 & 105 & 100 & 0 & 88 & 12 \\
\hline 34 & & & & & & 2 & 0.10 & 110 & 100 & 0 & 89 & 11 \\
\hline 35 & & & & & & 2 & 0.10 & 79 & 99 & 1 & 87 & 13 \\
\hline 36 & 5 & $32-64-20-1$ & 3,433 & 219,712 & 0.597 & 2 & 0.10 & 90 & 99 & 1 & 87 & 13 \\
\hline 37 & & & & & & 2 & 0.10 & 74 & 100 & 0 & 88 & 12 \\
\hline \multicolumn{13}{|c|}{... and later, for $\mathrm{NN}_{6}$ and Error Function $\mathrm{E} 2$} \\
\hline wtm & 6 & $32-32-5-2$ & 1,233 & 78,912 & 1.661 & 3 & 0.10 & 1,000 & 95 & 5 & --- & --- \\
\hline 39 & & & & & & 4 & 0.10 & 1,610 & 99.6 & 0.4 & --- & --- \\
\hline 40 & & & & & & & & 6,285 & 99.8 & 0.2 & --- & --- \\
\hline 41 & & & & & & 5 & 0.10 & 461 & 97.3 & 2.7 & --- & --- \\
\hline
\end{tabular}

Table 5: NN training and testing results.

\subsection{Comments on the Results}

Note first that all the trials achieved the immediate objective which was to demonstrate NNs learning to classify KPK positions. The choice of 3-layer networks throughout made this more likely. Because the initial 
state of the NN was randomly chosen, it was expected that only half the positions would initially be classified correctly and this was the case. However, the first few epochs of training typically improved accuracy rapidly.

All trials produced NNs with at least 92\% accuracy on the training set. For comparison, note that an NN configured to indicate that all wtm-positions were wins would be $76.5 \%$ accurate and an NN configured to indicate all btm-positions as wins for White would be $58.4 \%$ accurate (see Section 2). The difference between \%-accuracy achieved and \% of 'wins' was greatest for NNs trained on btm-positions.

The lowest success rates in trials 2 and 14 were due to the less rigorous error function E1 and the least challenging target of 0.15 . The use of the more rigorous error function E2 significantly improved the NNs' accuracy in classifying the positions of the training set and was used exclusively for the larger networks.

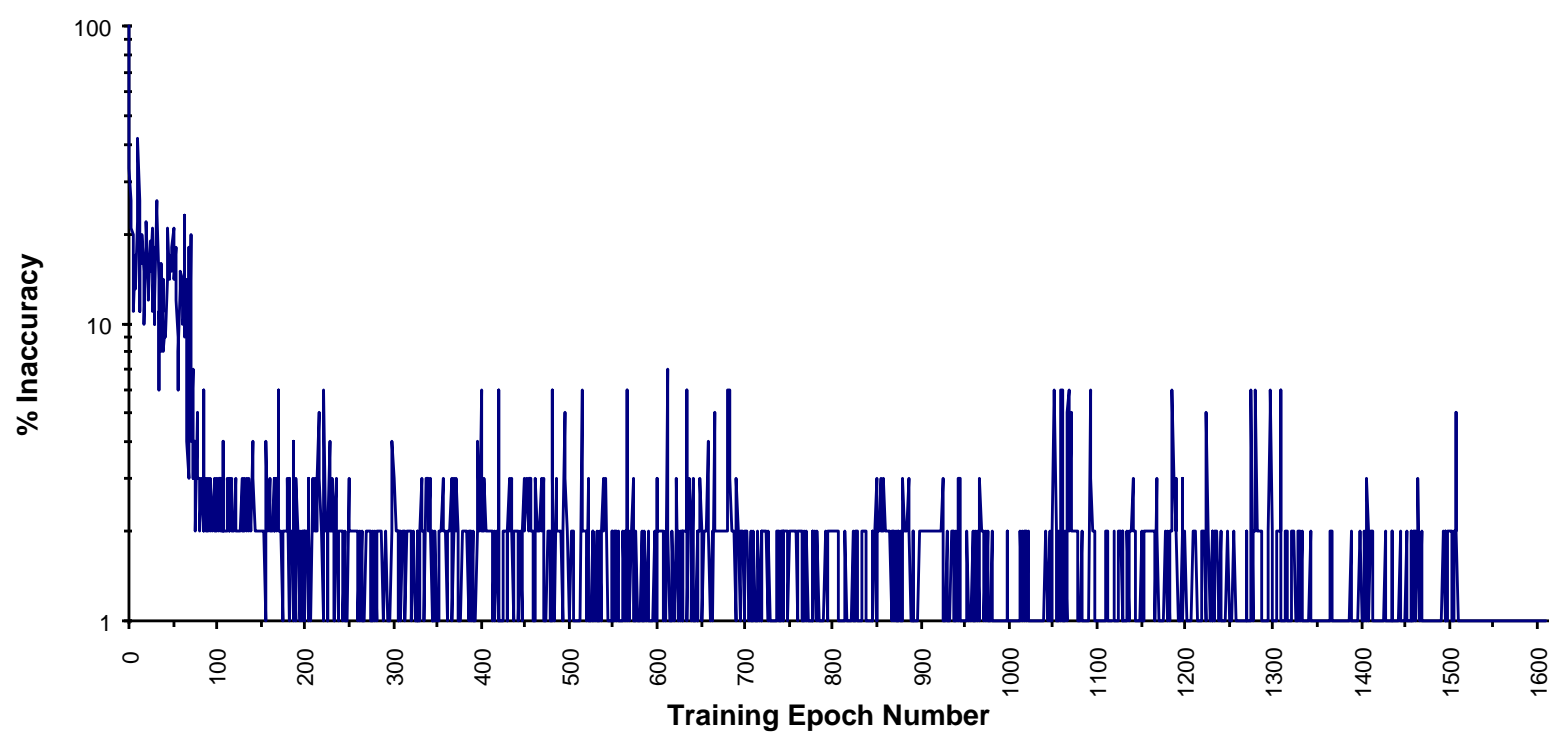

Figure 3: Residual inaccuracy of NN on a-file KPK wtm-positions.

The reduction in the percentage of misclassified positions for trial 39 (see Figure 3), shows that progress as measured by this criterion is far from monotonic. The NNs cited in Table 5 were not necessarily the final state of the NNs at the end of the training period.

The success rate on the 'new' testing-set positions was predictably lower than on the training-set positions. This is usually because the training set is not intrinsically characteristic of the testing set, or because the NN identifies too closely with the training set through overtraining. As positions were allocated at random to the small training and testing sets, the lower effectiveness on the testing set suggests overtraining. The slightly better performance on btm-positions is interesting and unexplained; it is not thought to have been caused by the slightly larger training set $\mathrm{T}_{2}$.

No clear pattern emerged as to what shape or size of NN was the best choice. The NNs with a compression factor of less than one did not prove conspicuously more effective. The last NN exercised on $F_{a}$ and $F_{d}$ certainly demonstrated that compression can be achieved as misclassified positions could be filed in a residual database and checked before the NN was consulted.

Trial 39 on the most difficult file subset $F_{a}$ left only 51 positions out of 20,643 misclassified. An examination of these positions in Table 6 is a salutary reminder that the NN has not been told about most aspects of chess, for instance, the rules of chess or the movement of pieces. Its essentially arithmetical/logical view of the positions has its limitations - but this is true of any static position evaluation function. Table 6 also indicates that some win-defining rules input as characteristics were not $100 \%$ effective (see \# 8, 17, 21, 22, 24). Therefore we see an argument for predefining subsets of the NN to force the effect of known rules.

Of the 51 misclassified positions, 42 are critical in that the result is affected by whose move it is. Eight are drawn with either wtm or btm (\# 1, 17, 21, 22, 24, 41, 49, 51) and one (\# 33) is a win with either side to move. 
Some positions translated one file to the right (e.g., \# 1) would have different values but most (e.g., 'wins' and \# 17, 21, 22, 24) would not. Positions \# 29 to 38 remind us that the wP is sometimes better on a2 than a3. The bK cannot move into check on b3, is forced to move to b2, and allows the wP to escape to a4. Neither the ' $\mathrm{a} 2 \rightarrow \mathrm{a} 4$ ' or the 'cannot move into check' rules are known to the NN. Nor is the fact that the board has only eight ranks and files. On reflection, it is surprising that the NN manages to classify the other 20,592 positions correctly.

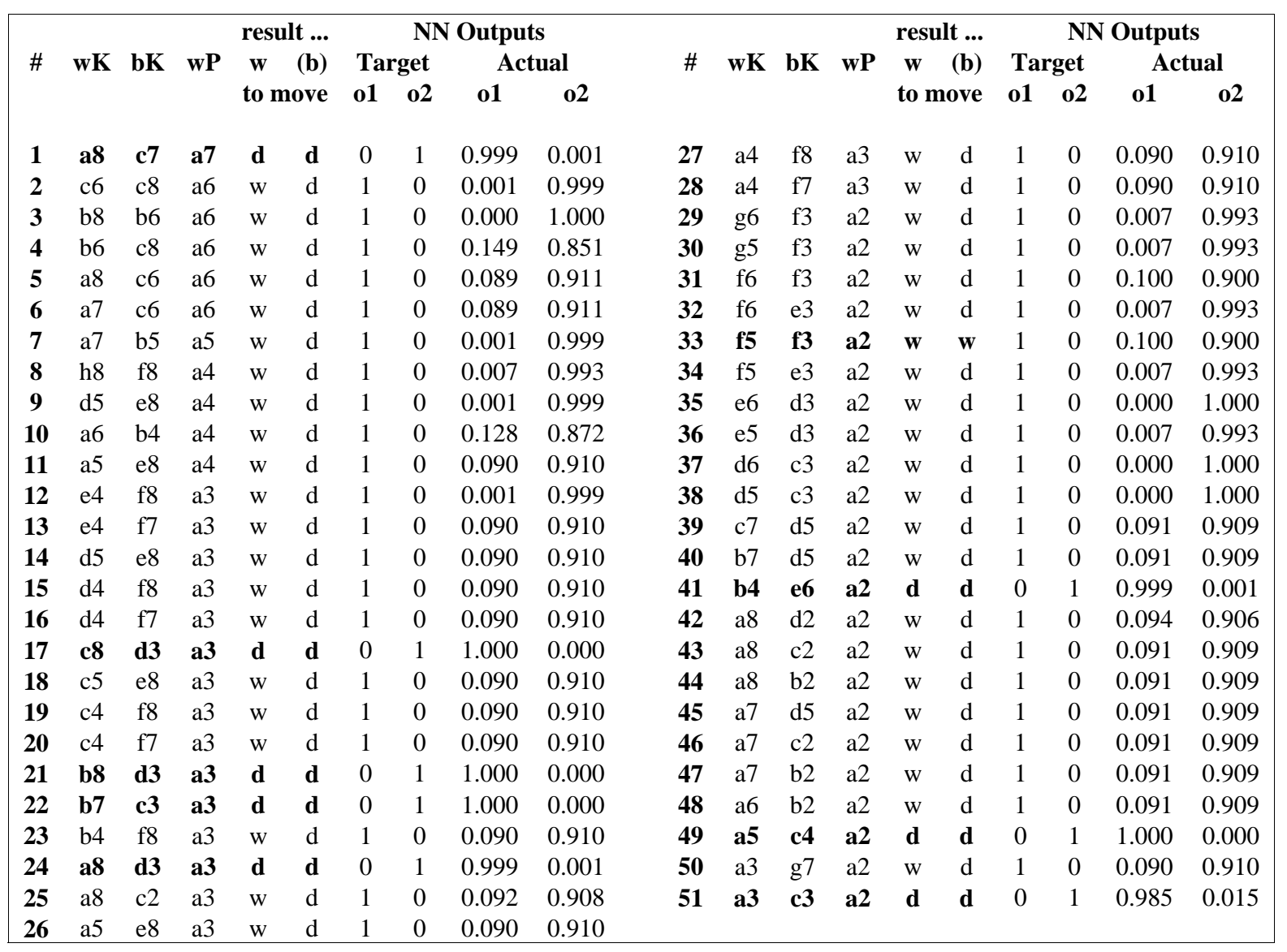

Table 6: The 51 misclassified ' $\mathrm{P}$ on the a-file' positions.

\section{THE EXPERIMENTAL TECHNIQUE AND FUTURE TASKS}

In the project as defined there was no time available to broaden the experimental goals or to finesse the experimental technique by following some tempting avenues of investigation which revealed themselves along the way. Other workers in this field may wish to take up the enquiry on some of the topics below. As discussed in Section 4, questions arose about characterising the positions, the NN-topology and training, and the chess implications of the results. Some examples are:

- What would 2-layer MLPs have achieved in comparison with 3-layer MLPs?

- What would an $n-n-1$ MLP have achieved on a Boolean coding of a KPK characterisation?

- Would the evolution of training/testing accuracy on $\mathrm{T}_{1 / 2} / \mathrm{S}_{1 / 2}$ have demonstrated over-training?

- How would training/testing accuracy for an NN vary as the size of training/testing sets increased?

- Which weights were most significant and which might have been pruned out to speed training?

- If NNs with initial weight values init and -init had been trained in parallel, would a comparison of their final values for weight $\mathrm{w}_{\mathrm{ijk}}$ have indicated whether that connection $\mathrm{n}_{\mathrm{ijk}}$ was significant?

- Would the NN-training concepts of momentum and annealing have contributed significantly? 
- How would MLPs have performed if tested on position value and depth instead of just value?

- How successfully would the MLPs have played as White (evaluating btm-positions) or Black (evaluating wtm-positions) against perfect play, success being measured in terms of \%-wins won and \%-of draws drawn?

- Would non-MLP NN topologies be applicable to the chess-endgame problem domain?

\section{THE NN CAPABILITY IN CONTEXT}

Rather than considering NN technology in isolation, let us ask how the 'NN approach' compares with the use of other technologies and whether NNs can be included in a hybrid approach to chess endgames.

Computer agents provide a mix of definitive, statistical and unqualified advice. Second-sourced endgame databases and optimal or correct programs are in the first category; Buro's (1995) Othello position evaluations are in the second. The NNs here are in the last category as they do not define the likelihood that their output is correct.

Reference has already been made to previous work (Beal and Clarke, 1980; Bramer, 1980) where the authors first had to recognise key features of positions by observation or received wisdom and then determined the sequence of use of these characteristics in a program code. The use of induction automation (Hunt and Stone, 1966; Quinlan, 1979; Shapiro and Niblett, 1982) promised to assist the latter activity but in fact they produced only a few results on chess endgames. The cause may be: (1) suitable position features were not used, (2) they were not used in the right combination, or (3) they do not exist at all.

Neural networks are dependent on the input characterisation of the positions but are free - almost too free - to combine these characteristics as encouraged by the target outputs. This suggests an experiment with a chosen position characterisation to compare the ease of construction, the efficiency and the effectiveness of:

- $\quad$ programs using the rules included in the characterisation;

- agents produced by rule-induction processes;

- agents using evaluation functions derived from Bayesian classification as in Buro (1995);

- a variety of NNs with a range of parameters including their compression factor.

Perhaps categorical data analysis (Buro, 1995) can further be used to initialise a 1-level NN which after training can in turn seed a 2-level NN as mentioned at the end of Section 3. NNs also offer the promise that, when trained, their most significant internal weights will reveal higher-level characteristics which are combinations of the input features. These characteristics may then enrich the original characterisation and after a further round of training, the first cycle of an evolutionary inductive process is complete. Then, any position features highlighted by NNs may also be input to programs, rule-induction processes or domain experts.

However, inferring new characteristics from weights is not easy and the best strategy may be to isolate them one at a time in subdomains where they are most conspicuous. Given that, in the last stages of an endgame, the winning side has accumulated some advantages by achieving some preliminary goals, it seems logical to investigate these feature-rich subdomains of the endgame first and - in a second example of a gradual approach - 'reach back' into the endgame, perhaps using move features as well as positional features. For example with $\mathrm{KPK}$, to win a position, White must achieve a subset of the following five objectives:

(1) defend the white Pawn from attack,

(2) pre-empt/loosen the black King's control of the Pawn's conversion square,

(3) clear a path for the white Pawn to run,

(4) run the Pawn to conversion to Queen or Rook, and

(5) mate the black King after the endgame conversion to KQK or KRK. 
This suggests that an NN trained on the closed sets $C_{p}$ or $C_{p} \cap F_{x}$ (see Section 2) for increasing $p$ and varying $x$ might pick up position features more easily one by one just as a human would. Other closed sets not used in this paper can be used to give a finer-grain approach to the full KPK domain.

There is however the danger that NNs will ascribe features to chess positions which are meaningful to NNs but not usable over the board by human players. Danger probably becomes likelihood if the input characterisation does not include the characteristics already known to the chess community.

Although this paper focuses on chess endgames, another natural question concerns the applicability of NNs in the opening phase of a game. Again there is a database of opening positions, compiled this time from play over the board. In the absence of definitive knowledge, each opening position may be assigned a 'probability' or 'likelihood' of winning based on past results. Domains with more smoothly varying probabilities are thought to be more amenable to NN modelling than endgames where the position value is $+1,0$ or -1 (Tesauro, 1992b, 1995). Existing linear position evaluation functions can serve as the start point for at least 2-level MLPs performing non-linear evaluations.

Given all the above, this section suggests a program of data mining on chess endgames and perhaps other areas of chess which will combine the best features of the various approaches today.

\section{SUMMARY}

The study reported here was triggered by a student project to demonstrate neural-network learning from a suitable dataset. The KPK endgame was chosen and a number of NNs were trained and tested: the immediate goal was achieved. The project raised a number of issues, particularly about the characterisation of the positions and the approach to the design of the best NNs to represent the original endgame database. It also raised questions about the relative merits of NNs and other data-mining techniques and their possible use in combination.

We propose a programme analysing chess data, particularly endgame databases, by using the available range of data-mining techniques and technologies. The purpose of the programme would be to create higher-level information and knowledge from the base data in the correct endgame databases. New knowledge will include both heuristics of good play and those perverse positions which are the 'exceptions to the rule' and by their singularity deserve to be enshrined as problems and studies.

We hope that Nunn's expectation of "more human friendly endgame explanations" will be fulfilled but expect that chess will not be reduced to a 32-man endgame with a slim 'how to win' pamphlet.

\section{ACKNOWLEDGEMENTS}

First of course, our thanks goes to Ken Thompson for his database work and particularly for providing us with one of his endgame CDs which included KPK. Our thanks go also to Don Beal, Jaap van den Herik and Dennis Breuker who all encouraged us in this initiative.

The web made available Steven Edwards' databases and John Tamplin's access service to Ken Thompson's databases. Finally, our thanks go to the referees for their constructive comments on the original content and presentation. 


\section{REFERENCES}

Beal, D.F. and Clarke, M.R.B. (1980). The Construction of Economical and Correct Algorithms for King and Pawn against King. Advances in Computer Chess 2 (ed. M.R.B. Clarke), pp. 1-30. Edinburgh University Press, Edinburgh. ISBN 0-85224-377-4.

Beale, R. and Jackson, T. (1990). Neural Computing: An Introduction. Institute of Physics Publishing, Bristol. ISBN 0-8527-4262-2.

Berliner, H. (1980). Computer Backgammon. Scientific American, Vol. 242, No. 6, pp. 54-62. ISSN 00368733.

Bramer, M.A. (1980). An Optimal Algorithm for King and Pawn against King using Pattern Knowledge. Advances in Computer Chess 2 (ed. M.R.B. Clarke), pp. 82-96. Edinburgh University Press, Edinburgh. ISBN 0-85224-377-4.

Bramer, M.A. (1982). Machine-Aided Refinement of Correct Strategies for the Endgame in Chess. Advances in Computer Chess 3 (ed. M.R.B. Clarke), pp. 93-112, esp. Appendix I. Pergamon Press, Oxford. ISBN 0-08026898-6.

Buro, M. (1995). Statistical Feature Combination for the Evaluation of Game Positions. Journal of Artificial Intelligence Research, Vol. 3, pp. 373-382. ISSN 1076-9757.

Clarke, M.R.B. (1977). A Quantitative Study of King and Pawn Against King. Advances in Computer Chess 1 (ed. M.R.B. Clarke), pp. 108-118. Edinburgh University Press, Edinburgh. ISBN 0-85224-292-1.

Edwards, S.J. (1994). Endgame Databases of Optimal Play to Mate. At publication time, this was to be found on the World Wide Web with ftp://chess.onenet.net/pub/chess/TB/ ... KPK.tbs/tbb/tbw

Fürnkranz, J. (1996). Machine Learning in Computer Chess: the Next Generation. ICCA Journal, Vol. 19, No. 3, pp. 147-161.

Hinde, C.J., Fletcher G.P., West A.A. and Williams, D.J. (1997). Neural Networks. ICL Technical Journal, Vol. 11 No. 2, pp. 244-278, esp. p. 259. ISSN 0142-1557.

Hunt, E.B., Marin, J. and Stone, P. (1966). Experiments in Induction. Academic Press. LCC 65-26400.

Messom, C.H. (1992). Engineering Reliable Neural Networks. Ph.D. thesis, Loughborough University, UK.

Michie, D. (1980). Chess with Computers. Interdisciplinary Science Reviews. Vol. 5, No. 3, pp. 215-227. ISSN 0308-0188.

Minsky, M.L. and Papert, S.A. (1969). Perceptrons, An Introduction to Computational Geometry. MIT Press, Cambridge, MA. Expanded edition (1988), MIT Press, Cambridge, Mass. ISBN 0-262-63111-3.

Nunn, J. (1992). Secrets of Rook Endings. B.T. Batsford, London. ISBN 0-7134-7164-6.

Nunn, J. (1994). Secrets of Pawnless Endings. B.T. Batsford, London. ISBN 0-7134-7508-0.

Nunn, J. (1995). Secrets of Minor-Piece Endings. B.T. Batsford, London. ISBN 0-7134-7727-X.

Quinlan, J.R. (1979). Discovering Rules by Induction from Large Collections of Examples. Expert Systems in the Micro-electronic Age. (ed. D. Michie), pp. 168-201. Edinburgh University Press, Edinburgh. ISBN 085224-381-2.

Rich, E. and Knight, K. (1991). Artificial Intelligence. Second Edition. McGraw-Hill Book Company, New York, N.Y. ISBN 0-07-100894-2. 
Roycroft, A.J. and Thompson, K.L. (1986). Queen and Pawn on a6 against Queen. Roycroft's 5-Men Chess Endgame Series, No. 2. Chess Endgame Consultants and Publishers, London.

Roycroft, A.J. and Beal, D.F. (1991). To Make Dumb Endgame Databases Speak. Advances in Computer Chess 6 (ed. D.F. Beal), pp. 149-159. Ellis Horwood Ltd., Chichester, England. ISBN 0-13-006537-4.

Rumelhart, D.E., Hinton, G.E. and Williams, R.J. (1987). Parallel Distributed Processing: Explorations in the Microstructure of Cognition, Vol. 1. MIT Press, Cambridge, Mass. ISBN 0-262-18120-7.

Samuel, A.L. (1959). Some Studies in Machine Learning using the Game of Checkers. IBM Journal of Research and Development, Vol. 3, No. 3, pp. 210-229. ISSN 0018-8646. Reprinted (1963) in Computers and Thought (eds. E.A. Feigenbaum and J. Feldman), pp. 71-105. McGraw-Hill Book Company, New York, N.Y. ISBN 07-020370-9.

Samuel, A.L. (1967). Some Studies in Machine Learning Using the Game of Checkers II Recent Progress. IBM Journal of Research and Development, Vol. 11, No. 6, pp. 601-617. ISSN 0018-8646. Reprinted (1970) in Human and Artificial Intelligence (ed. F.J. Crosson), pp. 81-116. Appleton-Century-Crofts, Educational Division, Meredith Corporation, New York, N.Y. ISBN 0-8919-7220-X.

Shapiro, A.D. and Niblett, T. (1982). Automatic Induction of Classification Rules for a Chess Endgame. Advances in Computer Chess 3 (ed. M.R.B. Clarke), pp. 73-92. Pergamon Press, Oxford. ISBN 0-08-0268986.

Sutton, R. S. and Barto, A. G. (1998). Reinforcement Learning: An Introduction. (esp. Section 11.1). MIT Press, Cambridge, Mass. ISBN 0-2621-9398-1.

Tesauro, G. (1992a). Practical Issues in Temporal Difference Learning. Machine Learning, Vol. 8, pp. 257277. ISSN 0885-6125.

Tesauro, G. (1992b). Temporal Difference Learning of Backgammon Strategy. Machine Learning: Proceedings of the 9th International Conference on Machine Learning 92 (eds. D. Sleeman and P. Edwards), pp. 451-457. Morgan Kaufmann, San Mateo, CA. ISBN 1-55860-247-X.

Tesauro, G. (1994). TD-Gammon, a Self-Teaching Backgammon Program, achieves Master-Level Play. Neural Computation, Vol. 6, No. 2, pp. 215-219. ISSN 0899-7667.

Tesauro, G. (1995). Temporal Difference Learning and TD-Gammon. Communications of the ACM, Vol. 38, No. 3, pp. 55-68. ISSN 0001-0782.

Thompson, K. (1986). Retrograde Analysis of Certain Endgames. ICCA Journal, Vol. 9, No. 3, pp. 131-139.

Walker, A.N. (1996). Hybrid Heuristic Search. ICCA Journal, Vol. 19, No. 1, pp. 17-23.

\section{POST-PUBLICATION NOTE}

This version corrects a few slips in or clarifies the content of the published version as follows:

- p212, Table 1, Set 5: 'Kb4/Kc5 are sub-optimal' for 'Kc5 (sub-opt.) or Kb4?' - see correction 4;

- p213, para 1, last line: ' $\mathrm{P}_{\mathrm{q}}$ ' for ' $\mathrm{P}_{7}$ ';

- p213, para 2, line 3: 'W( $\left.\mathrm{C}_{9}\right)$ ' for 'W(9)';

- p213, line -3: 'Kb4/Kc5 are sub-optimal' for 'Kb4 only draws' - Kb4 still wins;

- p214, Figure 1: ' $\mathrm{w}_{\mathrm{a} 10} \cdot \mathrm{X}_{\mathrm{a} 0}$ ' for ' $\mathrm{W}_{\mathrm{ab} 0} \cdot \mathrm{X}_{\mathrm{a} 0}$ ' on the arrow from $\mathrm{N}_{\mathrm{a} 0}$ to $\mathrm{N}_{11}$;

- p215, para 3, line 3: 'represent at most one of' for 'represent one of';

- p215, para 5: 'linear form' for 'linear equation';

- p216, Figure 2: the connector from $c_{24}$ to $c_{51}$ replaces that from $c_{22}$ to $c_{51}$ - see next correction;

- p217, Table 4, last row $\left(\mathrm{c}_{51}\right)$ : ' $\mathrm{x}_{18}$ ' for ' $\mathrm{x}_{16}$ ' - the check is that the wK and the wP are on different files. 